\title{
Influence of retinoblastoma-related gene silencing on the initiation of DNA replication by African cassava mosaic virus Rep in cells of mature leaves in Nicotiana benthamiana plants
}

\author{
Gareth Bruce ${ }^{2,3}$, Mei Gu${ }^{4}$, Nongnong Shi ${ }^{1}$, Yule Liü ${ }^{5}$ and Yiguo Hong ${ }^{1,2^{*}}$
}

\begin{abstract}
Background: Geminiviruses mainly infect terminally differentiated tissues and cells in plants. They need to reprogramme host cellular machinery for DNA replication. This process is thought to be mediated by inactivation of cell-cycle repressor proteins and by induction of host DNA synthesis protein expression through actions of the geminviral replication initiator protein (Rep).

Findings: Exploiting a Nicotiana benthamiana pOriz line, which is transformed with a transgene consisting of a direct repeat of the African cassava mosaic virus (ACMV)-replication origin (Ori) flanking a non-viral DNA region, and virus-induced RNA silencing (VIGS), the impact of host gene expression on replication of the ACMV-derived replicon was investigated. The ACMV Rep trans-replicated the viral episomal replicon in leaves of young but not older pOri2 plants. Upon VIGS-mediated down-regulation of N. benthamiana NbRBR1, the retinoblastoma-related protein gene coding for a negative cell-cycle suppressor, recovered the ability of ACMV Rep for trans DNA replication, whereas the silencing of NbPCNA coding for the sliding clamp of DNA polymerase had no effect.
\end{abstract}

Conclusions: These results suggest that the cellular machinery for DNA replication in differentiated tissues of older leaves cannot be reprogrammed by Rep alone but may need other uncharacterised viral and plant factors.

Keywords: African cassava mosaic virus (ACMV), DNA replication, Replication initiator protein (Rep), Retinoblastomarelated protein (RBR), Proliferating cell nuclear antigen (PCNA), Virus-induced RNA silencing (VIGS)

\section{Introduction}

African cassava mosaic virus (ACMV) is a singlestranded (ss) DNA virus in the genus Begomovirus, family Geminiviridae. ACMV possesses two circular DNAs, designated DNA-A and DNA-B of approximately $2.7 \mathrm{~kb}$ [1], both are required for systemic infection of plants [2]. DNA-A and DNA-B share an almost identical common region that contains cis-acting elements required for replication and transcriptional modulation of viral gene expression $[3,4]$. The bipartite ACMV genome encodes eight proteins that are responsible for the viral life cycle in and among host plants. The

\footnotetext{
* Correspondence: yiguo.hong@hznu.edu.cn

'Research Centre for Plant RNA Signalling, College of Life and Environmental Sciences, Hangzhou Normal University, Hangzhou 310036, China Full list of author information is available at the end of the article
}

multifunctional replication initiator protein (Rep) is essential for the initiation of rolling circle replication (RCR) of both DNA A and DNA B [5,6]. Rep also acts as a transcription repressor [7] and can trigger hypersensitive response and viral resistance in plants $[5,6,8,9]$. ACMV infection can induce antiviral RNA silencing defence [10], affect siRNA production [11], disturb microRNA biosynthesis and cause abnormal developmental phenotypes in plants $[12,13]$.

\section{Results and discussion}

An in planta trans-replication system [5] was utilised to investigate the impact of Nicotiana benthamiana retinoblastoma-related (NbRBR1) and proliferating cell nuclear antigen (NbPCNA) genes, (which code for a repressor of cell cycle and the sliding clamp of host DNA replicase,

\section{C) Biomed Central}


respectively), on viral DNA replication. The potato virus $\mathrm{X}$ (PVX)-based vector for virus induced RNA silencing (VIGS) was employed to knock-down NbRBR1 and NbPCNA expression. To achieve this, a 420-bp fragment corresponding to the 5' end of NbRBR1 (Genbank accession number: AY699399) was obtained through reversetranscription (RT)-PCR with total RNAs template from healthy $N$. benthamiana leaves using Qiagen RNeasy Minikit (Qiagen) for extraction and the primer pair PP369 (5’ ACGACATCGATATGGGTGGAGCTGAATAATTGTTC 3') and PP449 (5' CTCTTCCGGCC GTCTGAACCATACAGATTG 3'). The RT-PCR product was digested with ClaI/EagI and cloned into the same sites of the modified PVX/GFP vector to produce PVX/NbRBR1-GFP (Figure 1a). Similarly, the 5'-end 371-bp fragment of the N. benthamiana NbPCNA (Genbank accession number: AF303075) was obtained through RT-PCR using a pair of primers PP398 (5' TTTTCAATCGATATGTTGGGAATTACGGCTTGT 3') and PP450 (5' AATTACCGGCCGGTCACCGATTACTGCTAAGGT 3') to generate PVX/NbPCNA-GFP (Figure 1a). Correct insertions were confirmed by sequencing.

In each of three separate experiments, 3-4 young transgenic $N$. benthamiana line pOri-2 plants (25 days old) were inoculated with RNA transcripts for PVX/ GFP, PVX/NbRBR1-GFP or PVX/NbPCNA-GFP as previously described [9]. The transgenic pOri2 line contains a transgene consisting of a direct repeat of the ACMV replication origin (Ori) flanking a non-viral DNA region as previously described $[5,6]$. In contrast to mock-treated controls [plants were inoculated with RNase-free water] (Figure 1b), plants inoculated with recombinant PVX RNAs developed local chlorotic lesions at 4-6 days post-inoculation (dpi). Subsequently, mosaic and chlorosis appeared in the systemic young leaves at approximately $10 \mathrm{dpi}$, which were maintained in plants infected with PVX/GFP (Figure 1c). By contrast, plants systemically infected with PVX/NbRBR1-GFP or PVX/ NbPCNA-GFP started to show recovery from viral infection at approximately $14 \mathrm{dpi}$ and typical PVX symptoms disappeared almost completely at $25 \mathrm{dpi}$. In contrast, VIGS of NbRBR1 resulted in growth retardation, abnormal leaf development, and newly emerged leaves were irregularly shaped and had a definite curl downwards running the whole circumference of the leaf (Figure 1d). VIGS of NbPCNA also caused stunted growth and distorted leaves. Young leaves growing at the apical meristem were heavily crinkled, curled upwards and rosette in shape (Figure 1e). These phenotypic changes are similar to that previously described for suppression of $N b R B R 1$ or NbPCNA in $N$. benthamiana using various VIGS systems [14,15].

\section{a}

$\mathrm{PVX} / \mathrm{GFP}$

PVX/NbPCNA-GFP

PVX/NbRBR1-GFP
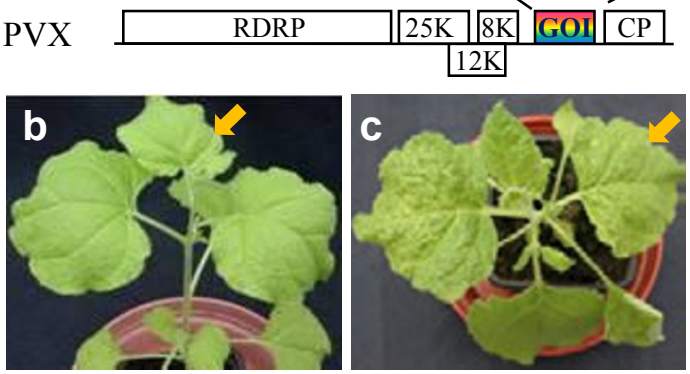

d
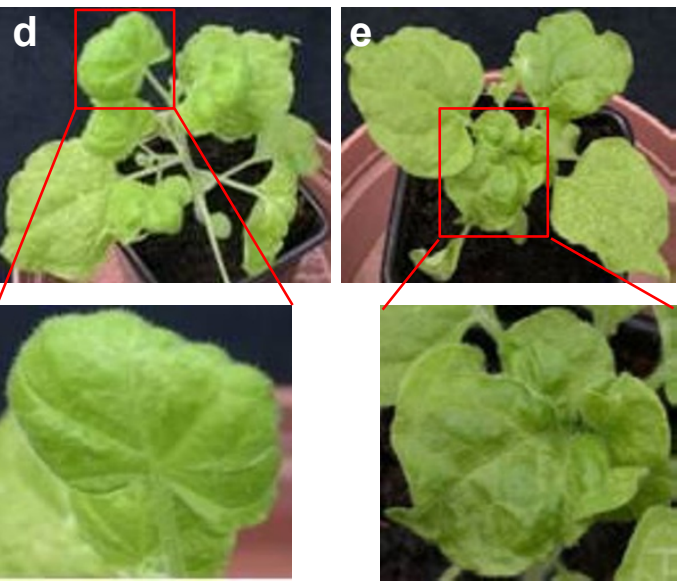

Figure 1 Virus induced RNA silencing (VIGS) (a) Construction of PVX/NbRBR1-GFP and PVX/NbPCNA-GFP in PVX/GFP. The PVX genome organisation is shown to encode the RNA-dependent RNA polymerase (RDRP), the triple-gene block ( $25 \mathrm{~K}, 12 \mathrm{~K}$ and $8 \mathrm{~K})$ and the capsid protein $(\mathrm{CP})$ as well as an insertion of gene of interest (GOI). (b-e) VIGS phenotypes of transgenic Nicotiana benthamiana line pOri2 plants. Plants (25 days old) were mock-inoculated (b) or inoculated with PVX/GFP (c), PVX/NbRBR1-GFP (d) or PVX/NbPCNAGFP (e). PVX/GFP infected plant showed mosaic and chlorosis at 10 days post-inoculation (dpi) whilst mock inoculated plant remained healthy. Plants challenged with either PVX/NbRBR1-GFP or PVX/ NbPCNA-GFP showed local and systemic infection at early stage (7$14 \mathrm{dpi}$ ) of viral invasion, but later on (approximately $21 \mathrm{dpi}$ onwards) newly growing leaves showed no viral symptoms. VIGS of NbRBR1 or NbPCNA caused abnormal leaf development and growth retardation. Plants (50 days old) were photographed at $25 \mathrm{dpi}$. The boxed portions of plants are enlarged to show VIGS-related phenotypes. Leaves of plants with mock inoculation (b) or infected with PVX/GFP (c) at the same developmental stage as these boxed leaves are indicated with an arrow. There leaves were infiltrated with agrobacterium and subsequently used for sqRT-PCR and PCR (see Figures 2 and 3).

VIGS of $N b R B R 1$ or $N b P C N A$ in pOri2 plants was further confirmed by semi-quantitative (sq) RT-PCR. Total RNAs (100 ng) were extracted at $31 \mathrm{dpi}$ from leaves at the age of 56-days old plants, and used as 
templates together with one of the two sets of primers PP369 and PP370 (5' TCTTGCGGCCGTCGCTTGTAG TACTTGCTTAAAAG 3') or PP398 and PP439 (5' TTGAGACGGCCGCACCTTCCTTTGTCGCAGAAA TTA 3') for sqRT-PCR assays. These primers allowed a specific detection of the 500-nt portion corresponding to the endogenous mRNA transcripts of the two target genes. Reduction in NbRBR1 (Figure 2a) or NbPCNA (Figure 2b) expression was obvious in the plants inoculated with PVX/NbRBR1-GFP or PVX/NbPCNA-GFP, respectively, when compared to that in mock-inoculated plants or plants infected with PVX/GFP. It was also noted that silencing of NbPCNA did not affect the level of NbRBR1 mRNA (Figure 2b). The equal amount of total RNAs in each of the sqRT-PCR reactions was verified by detecting a similar level of $18 \mathrm{~S}$ rRNA in all samples using primers PP271 (5'CGGCTACCACATC CAAGGAAGG 3') and PP272 (5' GAGCTGGAATTACCGCGGCTG 3') (Figure 2a, b). Taken together, these results demonstrate that VIGS of endogenous NbRBR1 and NbPCNA expression occurred and the aberrant phenotypes observed were caused by silencing of NbRBR1 or NbPCNA.

The effect of silencing of NbRBR1 or NbPCNA on the life cycle of ACMV, particularly in terms of DNA replication, was investigated in the pOri2-based trans-DNA replication system (Figure 3a). Trans-DNA replication to generate a circular episomal replicon occurs in the presence of ACMV Rep $[5,6]$. Mature leaves of healthy and PVX/GFP-infected pOri-2 plants, and leaves of pOri-2 plants which recovered from viral infection and showed phenotypic responses to NbRRB1 or NbPCNA silencing were infiltrated with Agrobacterium tumefaciens LBA4404 carrying pGreen0029/AC1234, pGreen0029/ AC1m2m3m4 or pGreen0029/ACm1m2m3m4 (Figure 3b). Expression ACMV complementary strand genes (CSG) including Rep (AC1) from these cassettes is under the control of the $35 \mathrm{~S}$ promoter and the CaMV polyadenylation signal. An equivalent amount of ACMV CSG RNA transcripts transcribed from/ AC1234, pGreen0029/AC1m2m3m4 or pGreen0029/ ACm $1 \mathrm{~m} 2 \mathrm{~m} 3 \mathrm{~m} 4$ was detected in agro-infiltrated leaf tissues by sqRT-PCR (unpublished data). Episomal circular DNA was readily detectable by PCR using primers P1 (5' TCGCGCTGATACCAGACGTTGC 3') and P2 (5' GGACTGGCATGAACTTCGGTG 3') (Figure 3a) and template DNA (100 ng) extracted from infiltrated leaf tissues at 6 days post agro-infiltration. Expression of Rep alone (pGreen0029/AC1m2m3m4) or together with AC2, AC3 and AC4 (pGreen0029/AC1234) in growing leaves of young healthy pOri2 plants at the age of 31 days, was able to initiate trans-replication. PCR (Figure 3c) detected a specific 1.6-kb fragment derived from episomal replica whilst no such DNA was detected in leaves infiltrated with pGreen $0029 / \mathrm{ACm} 1 \mathrm{~m} 2 \mathrm{~m} 3 \mathrm{~m} 4$ carrying agrobacteria as negative control, from which no ACMV gene product is translatable. At the older age (56 days), agro-infiltrated leaves of mock-, PVX/GFP, or PVX/NbPCNA-infected pOri2 plants were unable to trans-replicate the episome (Figure 3a). However, if NbRBR1 expression was reduced by VIGS (Figure 2a), circular episomes were readily detected (Figure $3 \mathrm{~d}$ ). Similar results were obtained in NbRBR1-silenced old leaves if Rep was expressed alone from pGreen0029/ AC1m $2 \mathrm{~m} 3 \mathrm{~m} 4$ (data not shown). These data suggest that the expressed ACMV Rep is only able to initiate DNA replication in older leaves if expression of the cell cycle block NbRBR1 is reduced.

Geminiviral Rep is not a DNA polymerase. However, during viral DNA replication the oligomeric Rep protein cleaves the viral replication origin TAATATT $\downarrow$ AC and acts as an ATP-dependent ligase to re-circularise progeny ssDNA $[3,16,17]$. Efficient viral DNA replication is also dependent on functional interplays between Rep

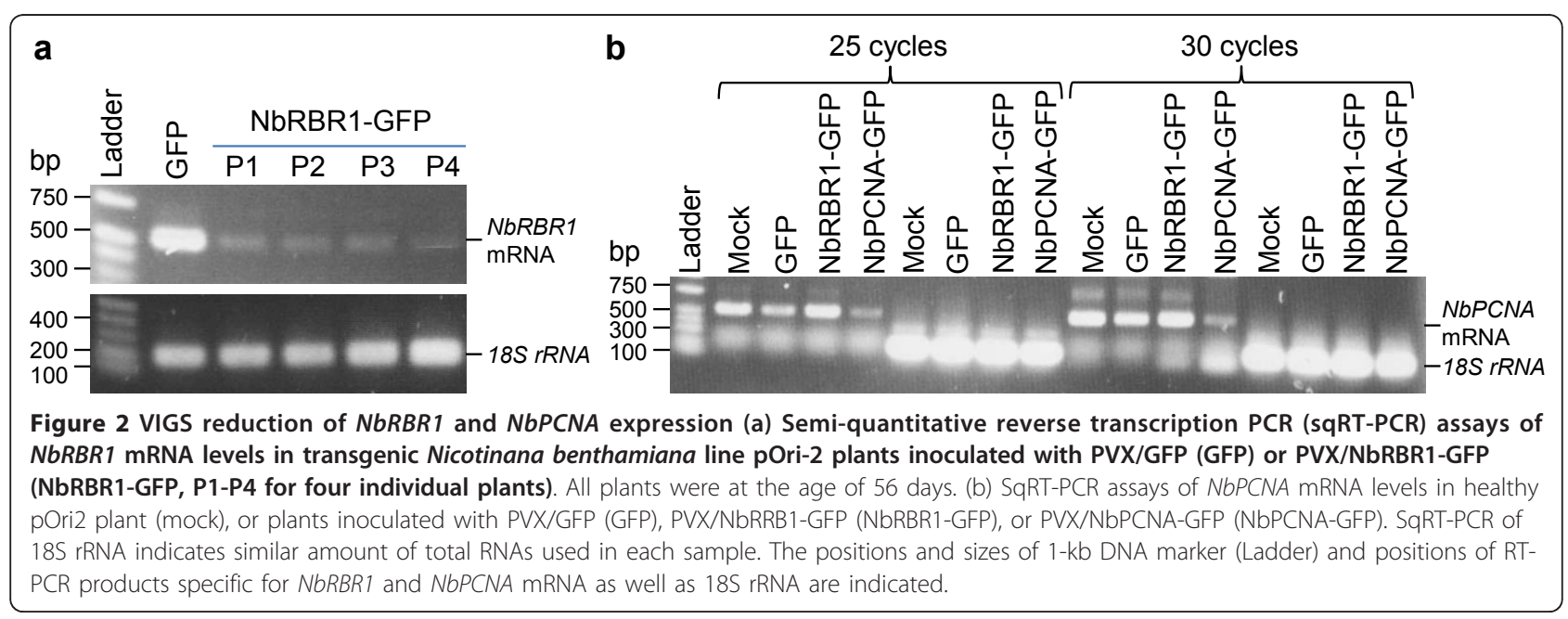




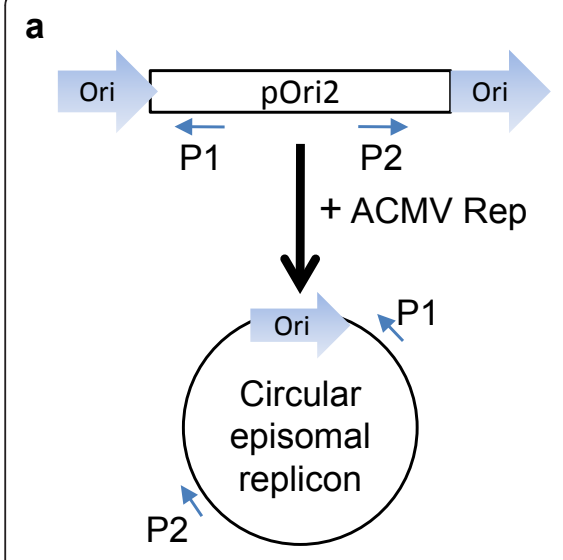

pOri2-based trans-DNA replication

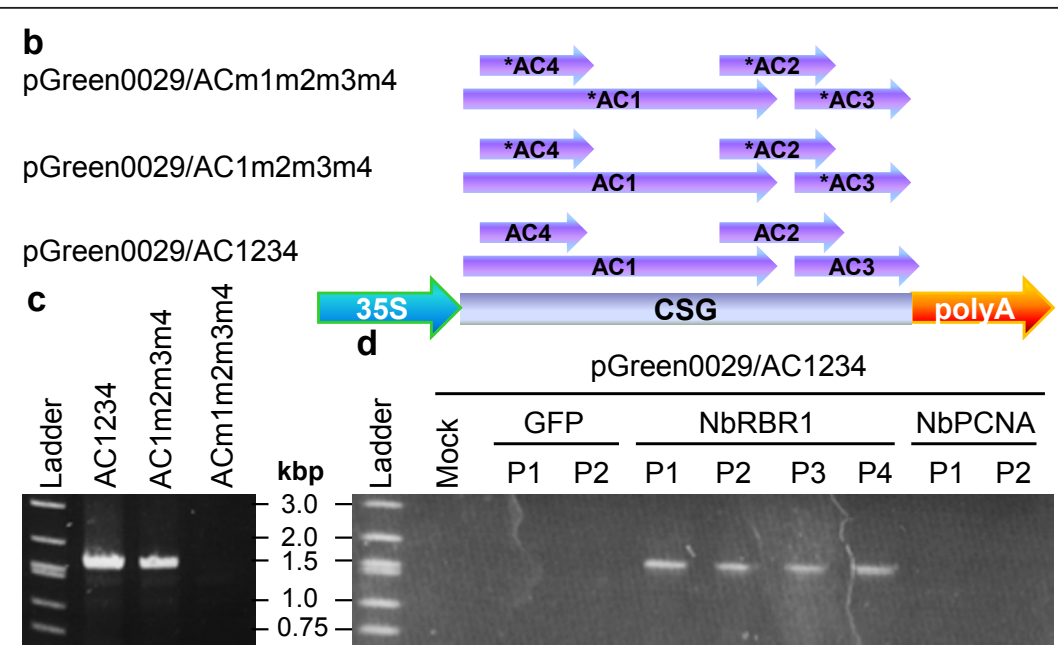

Figure 3 VIGS of NbRBR1 and NbPCNA affects episiomal DNA replication (a) Outline of the trans-DNA replication system in the transgenic pOri2 line [5,6]. The replication origin (Ori) of the African cassava mosaic virus (ACMV) and the P1/P2 primers for PCR detection of the circular episomal replicon in the presence of ACMV Rep are indicated. (b) Constructs for pGreen0029/AC1234, pGreen0029/AC1m2m3m4 and pGreen0029/ACm1m2m3m4 expressing the ACMV complementary-sense genes (CSG, AC1 - AC4], AC1 alone or no gene under the control of the CaMV 35 S promoter and polyA signal. Mutated genes are indicated by asterisks. (c) PCR assays for circular episomal replica in young N. benthamiana line pOri-2 plants at the age of 31 days. In three separate experiments, young growing leaves were infiltrated with Agrobacteria carrying pGreen0029/AC1234 (AC1234), pGreen0029/AC1m2m3m4 (AC1m2m3m4), or pGreen0029/ACm1m2m3m4 (ACm1m2m3m4). (d) Impact of VIGS-mediated reduction of NbRBR1 or NbPCNA expression on viral replication. In each of three experiments, line pOri2 plants (25 days old) were mock-inoculated (Mock), or inoculated with PVX/GFP (GFP, two plants P1-P2), PVX/NbRBR1-GFP (NbRBR1, four plants P1-P4) or PVX/ NbPCNA-GFP (NbPCNA, two plants P1-P2). At 25 days post-inoculation, leaves of these plants (50 days old) as indicated in Figure 1 were subsequently infiltrated with Agrabacteria carrying pGreen0029/AC1234 and analysed by sqRT-PCR (see Figure 2) and PCR 6 days later. The positions and sizes of 1-kb DNA marker (Ladder) are indicated.

and other viral proteins including the replication-enhancing protein and coat protein $[18,19]$, as well as with host factors such as replication factor C $[20,21]$. Moreover, geminiviruses infect terminally differentiated tissues/cells in which host DNA polymerases are not functional. Therefore, to establish infection, geminiviruses need to re-programme host cellular machinery for DNA replication. This process is thought to be mediated by inactivation of the cell-cycle repressor RBR protein through direct Rep-RBR interactions and by Rep-triggered induction of host DNA synthesis PCNA expression [22-25]. It is demonstrated that with the begomovirus Tomato golden mosaic virus, Rep-RBR interaction and PCNA accumulation are important for virus replication and infectivity in $N$. benthamiana $[22,24,26]$. On the other hand, in mastreviruses, such as Maize streak virus (MSV), an intact Rep RBR-interaction motif is not required for virus replication in culture cells or infectivity in maize, although it is possibly required for wild-type symptom development [27-29]. Indeed, wild-type MSV invades both vasculature and mesophyll cells of mature maize leaves. In contrast, MSV with a dysfunctional Rep RBR-interaction motif was restricted to the vasculature, in which dividing cells possess the active machinery for DNA synthesis. It is suggested that mature leaves contain high levels of RBR and the MSV
Rep-RBR interaction is essential only in tissues with high levels of active RBR [28]. However, prior to Repmediated deregulation of cell-cycle control to provide an environment that is able to accommodate replication, the Rep gene must be transcribed from double-stranded (ds) DNA intermediates to express mRNA from which the Rep protein can then be translated. It remains an open question how a geminivirus generates dsDNA from its ssDNA genomes in cells where the replication machinery is inactive.

ACMV Rep was unable to initiate RCR in cells of mature leaves of older pOri2 plants although it was effective in triggering RCR in cells of growing leaves of young plants. It is possible that young growing leaves possess many S-phase cells with an active DNA replication machinery, which ceases to function in cells of older mature leaves. One factor affecting the cellular DNA replication capacity can be different expression levels of genes that encode cell cycle modulators such as $R B R 1$. For instance, there may be low expression of $N b R B R 1$ in S-phase cells of growing leaves of young plants whereas in older leaves high NbRBR1 expression could effectively maintain cells in G1 phase with minimal replication activity. Indeed, in older tissues NbRBR1 mRNA was readily detectable (Figure 2). The ACMV Rep protein can hijack a functional replication apparatus 
in S-phase cells of young leaves to instigate and transreplicate the episome (Figure 3a). However, Rep alone, or together with other ACMV CSG products AC2, AC3 and AC4, cannot re-programme a "ceased" replication machinery through its interaction with RBR. On the other hand, it is not surprising that silencing of $N b P C N A$, an auxiliary protein of DNA polymerase, could not restore the deficiency of DNA replication (Figure 3d). However, reduction of NbRBR1 expression by VIGS may allow G1 cells of older leaves progressing into the $\mathrm{S}$ phase. Consequently, DNA synthesis functionality is re-activated and then exploited by ACMV Rep (Figure 3c-d). Thus, our findings suggest that some uncharacterised viral and/or plant factors may participate in reactivating the host cellular machinery for geminiviral DNA replication in terminally differentiated cells, and this process is a more complex one than previously proposed [22-25,30]. This idea is supported by the facts that the curtovirus $\mathrm{C} 4$ protein can induce plant cell cycle regulator gene expression [31] and that in fission yeast the ACMV Rep can affect cell division cycle despite no RBR homologue has been identified to date [32].

\section{Acknowledgements}

We thank D. C. Baulcombe for providing the original PVX vector. This project was supported in part by BBSRC grants (BBS/E/H/00032637; BBS/E/H/ 00YH221 to Y.H.). G. B. was supported by a BBSRC Studentship (BBS/S/K 2003/10157A to Y.H.). We are grateful to referees for critical comments and reading of the manuscript.

\section{Author details}

${ }^{1}$ Research Centre for Plant RNA Signalling, College of Life and Environmental Sciences, Hangzhou Normal University, Hangzhou 310036, China. ${ }^{2}$ Warwick HRI, University of Warwick, Wellesbourne, Warwick CV35 9EF, UK. ${ }^{3}$ Biological Sciences Research Unit, University of Glamorgan, Pontypridd, CF37 1DL Wales, UK. ${ }^{4}$ Clinical Sciences Research Institute, University of Warwick, Coventry CV2 2DX, UK. ${ }^{5}$ School of Life Sciences, Tsinghua University, Beijing 100084, China.

\section{Authors' contributions}

GB designed and performed experiments; MG, NS and YL contributed through discussion and revised paper. YH initiated the project, designed experiments and wrote paper. All authors read and approved the final manuscript.

\section{Competing interests}

The authors declare that they have no competing interests.

Received: 25 September 2011 Accepted: 28 December 2011 Published: 28 December 2011

\section{References}

1. Stanley J, Gay MR: Nucleotide-sequence of cassava latent virus-DNA. Nature 1983, 301:260-262.

2. Stanley J: Infectivity of the cloned geminivirus genome requires sequences from both dnas. Nature 1983, 305:643-645.

3. Stanley J: Analysis of african cassava mosaic-virus recombinants suggests strand nicking occurs within the conserved nonanucleotide motif during the initiation of rolling circle DNA-replication. Virology 1995, 206:707-712.

4. Hong YG, Saunders K, Stanley J: Transactivation of dianthin transgene expression by African cassava mosaic virus AC2. Virology 1997, 228:383-387
5. Hong YG, Stanley J, van Wezel R: Novel system for the simultaneous analysis of geminivirus DNA replication and plant interactions in Nicotiana benthamiana. J Virol 2003, 77:13315-13322.

6. Jin M, Li C, Shi Y, Ryabov E, Huang J, Wu Z, Fan Z, Hong Y: A single amino acid change in a geminiviral Rep protein differentiates between triggering a plant defence response and initiating viral DNA replication. J Gen Virol 2008, 89:2636-2641.

7. Hong YG, Stanley J: Regulation of african-cassava-mosaic-virus complementary-sense gene-expression by $\mathrm{N}$-terminal sequences of the replication-associated protein AC1. J Gen Virol 1995, 76:2415-2422.

8. Hong $Y$ G, Stanley J: Virus resistance in Nicotiana benthamiana conferred by African cassava mosaic virus replication-associated protein (AC1) transgene. Mol Plant-Microbe Interact 1996, 9:219-225.

9. Van Wezel $R$, Dong XL, Blake P, Stanley J, Hong YG: Differential roles of geminivirus Rep and AC4 (C4) in the induction of necrosis in Nicotiana benthamiana. Mol Plant Pathol 2002, 3:461-471.

10. Chellappan P, Vanitharani R, Ogbe F, Fauquet CM: Effect of temperature on geminivirus-induced RNA silencing in plants. Plant Physiol 2005, 138:1828-1841

11. Akbergenov R, Si-Ammour A, Blevins T, Amin I, Kutter C, Vanderschuren $H_{\text {, }}$ Zhang P, Gruissem W, Meins F, Hohn T, Pooggin MM: Molecular characterization of geminivirus-derived small RNAs in different plant species. Nucleic Acids Res 2006, 34:462-471.

12. Chellappan $P$, Vanitharani $R$, Fauquet $C M$ : MicroRNA-binding viral protein interferes with Arabidopsis development. Proc Natl Acad Sci USA 2005, 102:10381-10386.

13. Amin I, Patil BL, Briddon RW, Mansoor S, Fauquet CM: A common set of developmental miRNAs are upregulated in Nicotiana benthamiana by diverse begomoviruses. Virol J 2011, 8:143.

14. Jordan CV, Shen W, Hanley-Bowdoin LK, Robertson D: Geminivirus-induced gene silencing of the tobacco retinoblastoma-related gene results in cell death and altered development. Plant Mol Biol 2007, 65:163-175.

15. Peele C, Jordan CV, Muangsan N, Turnage M, Egelkrout E, Eagle P, HanleyBowdoin L, Robertson D: Silencing of a meristematic gene using geminivirus-derived vectors. Plant J 2001, 27:357-366.

16. Orozco BM, Hanley-Bowdoin L: Conserved sequence and structural motifs contribute to the DNA binding and cleavage activities of a geminivirus replication protein. J Biol Chem 1998, 273:24448-24456.

17. Pant V, Gupta D, Choudhury NR, Malathi VG, Varma A, Mukherjee SK: Molecular characterization of the Rep protein of the blackgram isolate of Indian mungbean yellow mosaic virus. J Gen Virol 2001, 82:2559-2567.

18. Malik PS, Kumar V, Bagewadi B, Mukherjee SK: Interaction between coat protein and replication initiation protein of Mung bean yellow mosaic India virus might lead to control of viral DNA replication. Virology 2005, 337:273-283.

19. Settlage SB, See RG, Hanley-Bowdoin L: Geminivirus C3 protein: replication enhancement and protein interactions. J Virol 2005, 79:9885-9895.

20. Luque A, Sanz-Burgos A, Ramirez-Parra E, Castellano MM, Gutierrez C: Interaction of geminivirus rep protein with replication factor $C$ and its potential role during geminivirus DNA replication. Virology 2002, 302:83-94.

21. Settlage SB, Miller AB, Gruissem W, Hanley-Bowdoin L: Dual interaction of a geminivirus replication accessory factor with a viral replication protein and a plant cell cycle regulator. Virology 2001, 279:570-576.

22. Ach RA, Durfee T, Miller AB, Taranto P, HanleyBowdoin L, Zambryski PC, Gruissem W: RRB1 and RRB2 encode maize retinoblastoma-related proteins that interact with a plant D-type cyclin and geminivirus replication protein. Mol Cellular Biol 1997, 17:5077-5086.

23. Bagewadi B, Chen SJ, Lal SK, Choudhury NR, Mukherjee SK: PCNA interacts with Indian mung bean yellow mosaic virus rep and downregulates rep activity. J Virol 2004, 78:11890-11903.

24. Egelkrout EM, Robertson D, Hanley-Bowdoin L: Proliferating cell nuclear antigen transcription is repressed through an E2F consensus element and activated by geminivirus infection in mature leaves. Plant Cell 2001, 13:1437-1452.

25. Nagar S, Pedersen TJ, Carrick KM, Hanleybowdoin L, Robertson D: A geminivirus induces expression of a host dna-synthesis protein in terminally differentiated plant-cells. Plant Cell 1995, 7:705-719.

26. Kong KL, Orozco BM, Roe JL, Nagar S, Ou S, Feiler HS, Durfee T, Gruissem W, Robertson D, Hanley-Bowdoin L: A geminivirus replication protein interacts with the retinoblastoma protein through a novel 
domain to determine symptoms and tissue specificity of infection in plants. EMBO J 2000, 19:3485-3495.

27. Shepherd DN, Martin DP, McGivern DR, Boulton MI, Thomson JA, Rybicki EP: A three-nucleotide mutation altering the Maize streak virus Rep pRBRinteraction motif reduces symptom severity in maize and partially reverts at high frequency without restoring pRBR-Rep binding. J Gen Virol 2005, 86:803-813.

28. McGivern DR, Findlay KC, Montague NP, Boulton MI: An intact RBR-binding motif is not required for infectivity of Maize streak virus in cereals, but is required for invasion of mesophyll cells. J Gen Virol 2005, 86:797-801.

29. Shepherd DN, Martin DP, Varsani A, Thomson JA, Rybicki EP, Klump HH: Restoration of native folding of single-stranded DNA sequences through reverse mutations: an indication of a new epigenetic mechanism. Arch Biochem Biophys 2006, 453:106-120.

30. Hanley-Bowdoin L, Settlage SB, Orozco BM, Nagar S, Robertson D: Geminiviruses: Models for plant DNA replication, transcription, and cell cycle regulation. Crit Rev Plant Sci 1999, 18:71-106.

31. Lai J, Chen H, Teng K, Zhao Q, Zhang Z, Li Y, Liang L, Xia R, Wu Y, Guo H, Xie Q: RKP, a RING finger E3 ligase induced by BSCTV C4 protein, affects geminivirus infection by regulation of the plant cell cycle. Plant J 2009, 57:905-917.

32. Kittelmann K, Rau P, Gronenborn B, Jeske H: Plant geminivirus Rep protein induces rereplication in fission yeast. J Virol 2009, 83:6769-6778.

doi:10.1186/1743-422X-8-561

Cite this article as: Bruce et al:: Influence of retinoblastoma-related gene silencing on the initiation of DNA replication by African cassava mosaic virus Rep in cells of mature leaves in Nicotiana benthamiana plants.

Virology Journal 2011 8:561.

\section{Submit your next manuscript to BioMed Central and take full advantage of:}

- Convenient online submission

- Thorough peer review

- No space constraints or color figure charges

- Immediate publication on acceptance

- Inclusion in PubMed, CAS, Scopus and Google Scholar

- Research which is freely available for redistribution

Submit your manuscript at www.biomedcentral.com/submit 